\title{
Image of the transverse bunch profile via coherent optical transition radiation
}

\author{
A. Potylitsyn, ${ }^{1,3,}$ L. Sukhikh, ${ }^{1}$ T. Gusvitskii $\odot,{ }^{1}$ G. Kube $\odot,{ }^{2,3}$ and A. Novokshonov ${ }^{2}$ \\ ${ }^{1}$ National Research Tomsk Polytechnic University (TPU), Tomsk, 634050, Russia \\ ${ }^{2}$ Deutsches Elektronen-Synchrotron (DESY), Hamburg, 22607, Germany \\ ${ }^{3}$ National Research Nuclear University (MEPhI), Moscow, 115409, Russia
}

(Received 27 December 2019; accepted 15 April 2020; published 27 April 2020)

\begin{abstract}
The use of optical transition radiation (OTR) monitors is a standard technique to measure transverse beam profiles at many electron accelerators. With modern accelerator technology it is possible to produce and accelerate even ultrashort electron bunches with sub-femtosecond duration. Such bunches interacting with the OTR target generate coherent optical transition radiation (COTR). For the COTR case, a reconstruction of the bunch profile from a recorded image using a conventional optical scheme is a task with inconclusive solution. In this paper an approach is proposed which is based on the strict propagation of COTR fields through a focusing lens. As result a linear dependence of the measured rms image size on the bunch size is obtained.
\end{abstract}

DOI: 10.1103/PhysRevAccelBeams.23.042804

\section{INTRODUCTION}

Optical transition radiation (OTR) monitors are widely used for transverse profile measurements of relativistic electron beams [1-4]. The authors of Ref. [5] have shown that such a technique can provide a submicron spatial resolution, using the so-called "point spread function (PSF) dominated regime". In order to take into account the parameters of a real optical system, an approach was developed in Ref. [6] based on the OTR characteristics using the commercial ray tracing program Zemax Optic Studio ${ }^{(0)}$ [7]. However, it should be noted that OTR monitors are able to measure transverse beam profiles only for incoherent radiation. In other words, bunch lengths or the scale of inhomogeneities have to be much longer than the OTR wavelength of observation.

Modern accelerator technologies (laser-driven plasma accelerators or free-electron lasers) [8-10] allow us to generate bunches with sub-femtosecond and even attosecond bunch lengths. Evidently, radiation emitted from such ultra-short bunches in the visible spectral region becomes coherent. Therefore, conventional OTR based diagnostic techniques cannot be applied any more and new diagnostic approaches are required [11]. In the case of coherent emission, the radiation intensity depends on the

"potylitsyn@tpu.ru

Published by the American Physical Society under the terms of the Creative Commons Attribution 4.0 International license. Further distribution of this work must maintain attribution to the author(s) and the published article's title, journal citation, and DOI. square of the number of electrons inside the bunch, and the spectral-angular distribution of coherent optical transition radiation (COTR) is determined by the one of conventional incoherent OTR and the bunch form factor [12]. A profile image generated from a bunch emitting COTR and measured with a standard optical system consisting of a focusing lens would look like a ring structure with a deep central minimum [13]. This is in contrast to a conventional OTR image which is formed by averaging the OTR intensities from each individual electron over all electrons inside the bunch, thus representing a replica of the original transverse bunch shape. One exception is the case when the transverse bunch size is in the order of not more than a few OTR wavelengths. In this situation the measured bunch image is determined by the single particle OTR field resulting in a central dip in the intensity distribution, see, e.g., Ref. [5].

A few approaches were developed in order to reconstruct bunch profiles based on COTR and a conventional OTR monitor scheme as depicted in Fig. 1 [13-16]. However, the approximations in use were rough, and detailed simulations of the process were troublesome. In the present work a consistent description of the COTR based imaging process will be presented which allows to connect image parameters and the transverse bunch size.

\section{MODEL FOR OTR BASED IMAGING}

The present model is based on a standard optical scheme as depicted in Fig. 1. To simplify the final expressions describing the radiation pattern in the detector plane and taking into account an initial beam profile, dimensionless variables will be used in accordance with Ref. [17]: 


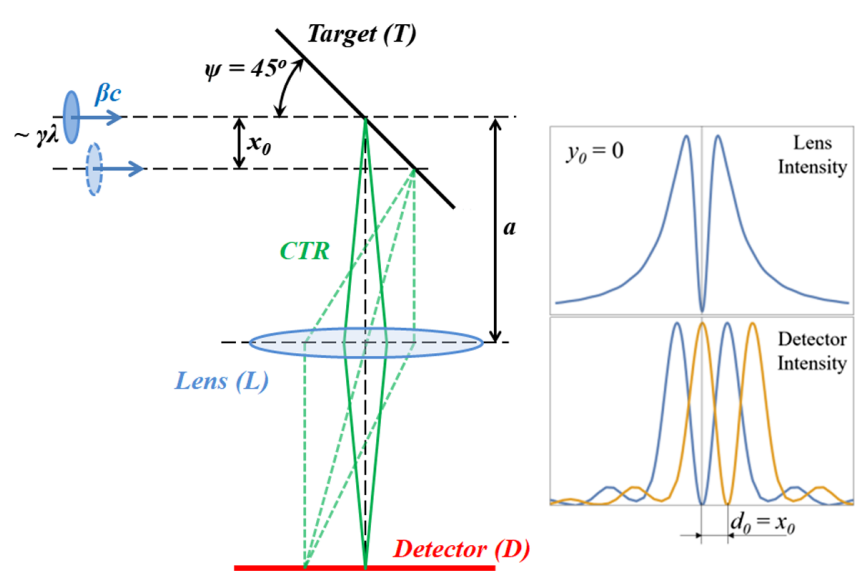

FIG. 1. Sketch of OTR beam profile monitor scheme.

$$
\begin{aligned}
\left\{x_{T}, y_{T}\right\} & =\frac{2 \pi}{\gamma \lambda}\left\{X_{T}, Y_{T}\right\} \\
\left\{x_{L}, y_{L}\right\} & =\frac{\gamma}{a}\left\{X_{L}, Y_{L}\right\} \\
\left\{x_{D}, y_{D}\right\} & =\frac{2 \pi}{\gamma \lambda}\left\{X_{D}, Y_{D}\right\} .
\end{aligned}
$$

Here $\gamma$ is the Lorentz factor, $\lambda$ the wavelength of observation, and $a$ the distance between target and a lens. Cartesian coordinates in Eq. (1) indicated by small letters $\left\{x_{i}, y_{i}\right\}$ are dimensionless ones, by capital letters $\left\{X_{i}, Y_{i}\right\}$ dimensioned ones, and the indices $i=T, L, D$ correspond to target (T), lens (L) and detector (D) plane. All coordinates are defined in frames connected with the optical axes of the system, see Fig. 1.

In the limit of ultrarelativistic electron energies $\gamma \gg 1$ the OTR process is considered as the reflection of the electron field by a perfectly conducting target (an ideal mirror). For a particle crossing the target with trajectory coordinates $\left\{x_{0}, y_{0}\right\}$ relative to the optical axes (impact parameter $\left.\sqrt{x_{0}^{2}+y_{0}^{2}}\right)$, it is straightforward to derive the fields in the detector plane in paraxial approximation, see, e.g., Ref. [17]:

$$
\begin{aligned}
E_{\{x, y\}}^{D}\left(x_{D}, y_{D}, x_{0}, y_{0}\right) \\
=\operatorname{const} \int \mathrm{d} x_{T} \mathrm{~d} y_{T} \frac{\left\{x_{T}-x_{0}, y_{T}-y_{0}\right\}}{\sqrt{\left(x_{T}-x_{0}\right)^{2}+\left(y_{T}-y_{0}\right)^{2}}} \\
\quad \times \mathrm{K}_{1}\left(\sqrt{\left(x_{T}-x_{0}\right)^{2}+\left(y_{T}-y_{0}\right)^{2}}\right) \exp \left[i \frac{x_{T}^{2}+y_{T}^{2}}{4 \pi R}\right] \\
\quad \times 4 \frac{\sin \left[x_{m}\left(x_{T}+\frac{x_{D}}{M}\right)\right]}{x_{T}+\frac{x_{D}}{M}} \times \frac{\sin \left[y_{m}\left(y_{T}+\frac{y_{D}}{M}\right)\right]}{y_{T}+\frac{y_{D}}{M}} .
\end{aligned}
$$

Here $\mathrm{K}_{1}$ is the modified Bessel function, $R=\frac{a}{\gamma^{2} \lambda},\left\{x_{m}, y_{m}\right\}$ is the aperture of the lens (for simplicity with squared shape), $M$ is the optical system magnification, and dimensioned constants are condensed in the amplitude factor

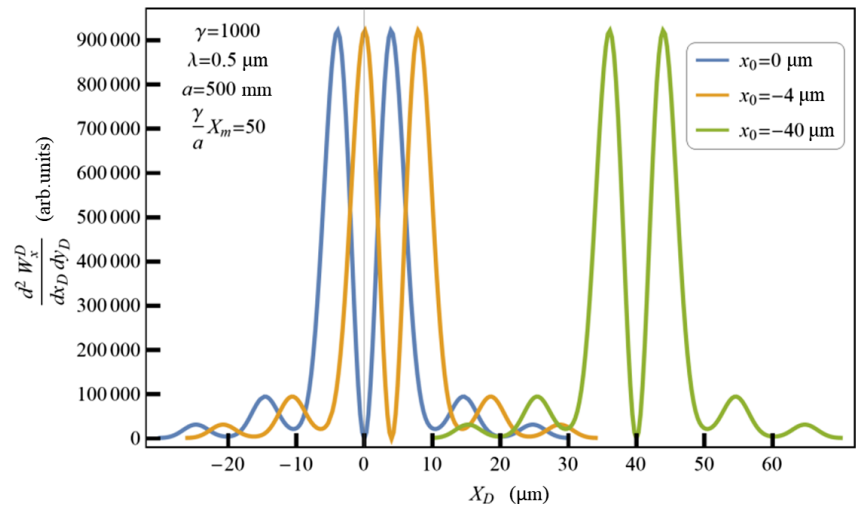

FIG. 2. OTR image distributions generated from electrons with different impact parameters.

const. The integration in Eq. (2) is performed over the target surface $\left(-x_{T_{\max }}<x_{T}<x_{T_{\max }},-y_{T_{\max }}<y_{T}<y_{T_{\max }}\right)$.

Evidently, the particle displacement relative to the optical axis (or relative to the target center) is much less than the corresponding target size. In order to diminish the calculation time for the integration in Eq. (2), the integration limits were chosen to be $x_{T_{\max }}=y_{T_{\max }}=5$. It follows that Eq. (2) can be applied for $\left|x_{0}\right|,\left|y_{0}\right|<1$ with good accuracy. In dimension units, this corresponds to $X_{T_{\max }}=$ $Y_{T_{\max }}=400 \mu \mathrm{m},\left|x_{0}\right|,\left|y_{0}\right|<80 \mu \mathrm{m}$, assuming $\gamma=1000$ and $\lambda=0.5 \mu \mathrm{m}$.

The OTR intensity distribution generated by a particle with impact parameter $\left\{x_{0}, y_{0}\right\}$ in the detector plane is calculated according to

$$
\frac{\mathrm{d}^{2} W_{0\{x, y\}}^{D}}{\mathrm{~d} x_{D} \mathrm{~d} y_{D}}=\text { const } \times\left|E_{\{x, y\}}^{D}\left(x_{D}, y_{D}, x_{0}, y_{0}\right)\right|^{2} .
$$

Figure 2 illustrates the difference between OTR based electron images with $x_{0}=0$ and $x_{0} \neq 0$. The following parameters were used for the calculation: $\gamma=1000$, $\lambda=0.5 \mu \mathrm{m}, \quad a=500 \mathrm{~mm}, M=1, X_{m}=Y_{m}=25 \mathrm{~mm}$ (corresponding to an angular lens aperture of $50 \gamma^{-1}$ ). For the magnification $M=1$ the shift of the OTR intensity minimum is equal to the impact parameter $x_{0}$, for $M \neq 1$ it is simply scaled. It should be emphasized that the pattern shape does not depend on the impact parameter and can be described by the universal dependence

$$
\frac{\left.\mathrm{d}^{2} W_{0}^{D} x_{0}, y_{0}\right\}}{\mathrm{d} x_{D} \mathrm{~d} y_{D}}=\operatorname{PSF}\left(x_{D}-x_{0}, y_{D}-y_{0}\right)
$$

with PSF the so-called point spread function (PSF) or, in other words, the response of the monitor optical system to a point charge crossing the target. Equation (4) is the basis to extract beam size information from a measured OTR intensity distribution in the detector plane, the intensity distribution represents the convolution of the PSF with the transverse beam profile. 


\section{COHERENT OTR EMISSION}

As pointed out before the image generation in case of incoherent OTR can be interpreted as PSF convolution with the bunch profile. For COTR it is not the intensity but the particle field which has to be considered. Therefore in the following an expression for this field will be derived. For the sake of simplicity henceforth a bunch with longitudinal Gaussian distribution is considered:

$$
\rho\left(x_{b}, y_{b}, z_{b}\right)=\rho\left(x_{b}, y_{b}\right) \frac{1}{\sqrt{2 \pi} \sigma_{z}} \exp \left\{-\frac{z_{b}^{2}}{2 \sigma_{z}^{2}}\right\} .
$$

Here $\sigma_{z}$ represents the longitudinal $\mathrm{rms}$ bunch length and $\rho\left(x_{b}, y_{b}\right)$ the transverse distribution of the electrons inside the bunch (as a rule the 2-D gaussian distribution with rms sizes $\sigma_{x}, \sigma_{y}$ ). In the present case, instead of the two-fold integration in Eq. (2) a five-fold integration over target surface and bunch volume has to be performed:

$$
\begin{aligned}
& E_{\text {coh }\{x, y\}}^{D}\left(x_{D}, y_{D}, \sigma_{x}, \sigma_{y}\right) \\
& =\text { const } \int \mathrm{d} x_{T} \mathrm{~d} y_{T} \mathrm{~d} x_{b} \mathrm{~d} y_{b} \mathrm{~d} z_{b} \rho\left(x_{b}, y_{b}, z_{b}\right) \\
& \quad \times \frac{\left\{x_{T}-x_{b}, y_{T}-y_{b}\right\}}{\sqrt{\left(x_{T}-x_{b}\right)^{2}+\left(y_{T}-y_{b}\right)^{2}}} \\
& \quad \times K_{1}\left(\sqrt{\left(x_{T}-x_{b}\right)^{2}+\left(y_{T}-y_{b}\right)^{2}}\right) \\
& \quad \times \exp \left\{i \frac{x_{T}^{2}+y_{T}^{2}}{4 \pi R}\right\} 4 \frac{\sin \left[x_{m}\left(x_{T}+\frac{x_{D}}{M}\right)\right]}{x_{T}+\frac{x_{D}}{M}} \frac{\sin \left[y_{m}\left(y_{T}+\frac{y_{D}}{M}\right)\right]}{y_{T}+\frac{y_{D}}{M}} .
\end{aligned}
$$

Solving Eq. (6) by numerical integration it is possible to calculate the COTR pattern in the detector plane

$$
\begin{aligned}
& \frac{\mathrm{d}^{2} W_{\operatorname{coh}\{x, y\}}^{D}\left(x_{D}, y_{D}, \sigma_{x}, \sigma_{y}\right)}{\mathrm{d} x_{D} \mathrm{~d} y_{D}} \\
& \quad=\mathrm{const}\left|E_{\operatorname{coh}\{x, y\}}^{D}\left(x_{D}, y_{D}, \sigma_{x}, \sigma_{y}\right)\right|^{2} .
\end{aligned}
$$

In order to reduce the numerical effort the integration over $z$ can be removed if the COTR pattern is measured for a narrow spectral bandwidth $\Delta \lambda / \lambda \ll 1$. In this case the $z$-integration is reduced to a multiplication by $\exp \left\{-4 \pi^{2} \lambda^{2} / \sigma_{z}^{2}\right\}$. However, even after this simplification the remaining four-fold integration is rather cumbersome and requires a large amount of computing power.

For further simplification, in the following the contribution from a single particle will be considered, i.e., $\rho\left(x_{b}, y_{b}\right)=q \delta\left(x_{b}-x_{0}, y_{b}-y_{0}\right)$ with $q$ the particle charge. Real and imaginary parts of the field in the detector plane are calculated according to Eq. (6) [or Eq. (2) which is the identical procedure for a single particle] and shown in Fig. 3. The comparison shows that the imaginary part $\Im\left[E_{x}^{D}\right]$ is about a factor 1000 smaller than the real one $\Re\left[E_{x}^{D}\right]$. Hence $\Im\left[E_{x}^{D}\right]$ is omitted in the intensity calculation hereafter. Furthermore, in order to speed up the computational time
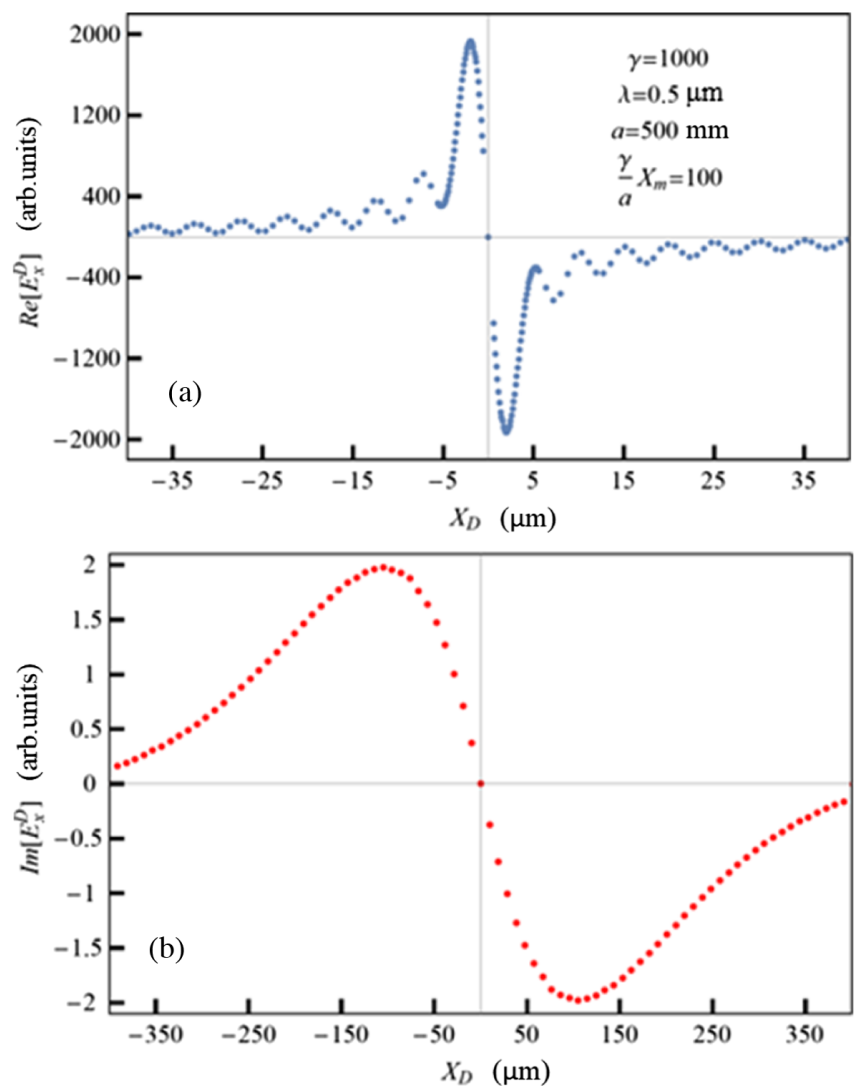

FIG. 3. (a) Real and (b) imaginary part of the single particle OTR field in the detector plane according to Eq. (2). The parameter set used for the calculation is shown in the insert of (a), the magnification of the optical system here and in the further course of the work is $M=1$.

in the following the real component is treated with a numerical accuracy of only better than $10^{-5}$.

In order to investigate the influence of the lens aperture, Fig. 4 shows calculations of $\Re\left[E_{x}^{D}\right]$ for a single particle according to Eq. (2) for two different aperture sizes

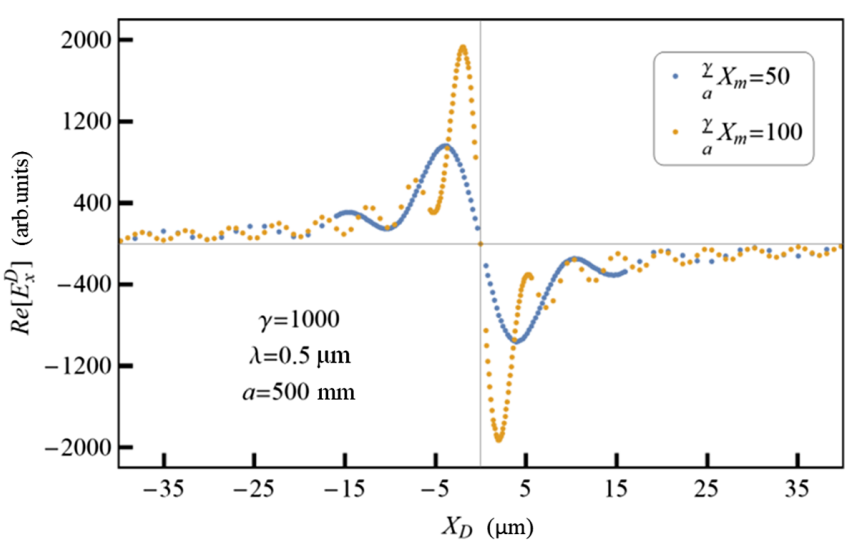

FIG. 4. Comparison of the real parts of the OTR field in the detector plane using two different lens apertures. The parameter set used for the calculation is shown in the insert. 


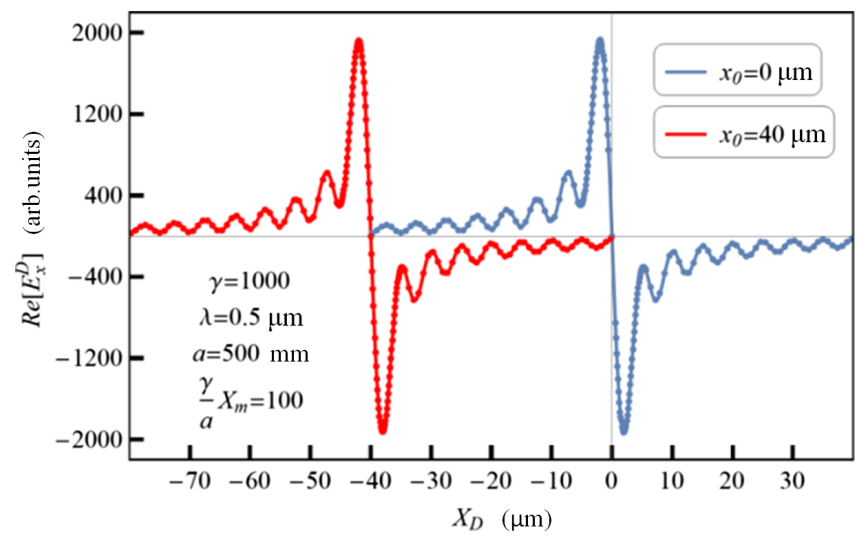

FIG. 5. Real part of the single particle OTR field in the detector plane for two different impact parameters. The parameter set used for the calculation is shown in the insert.

$x_{m} / y_{m}=50$ and 100. As expected from classical diffraction theory, an increase in the lens aperture results in a narrowing of the field extension and therefore in an improved resolution.

Fig. 5 shows a comparison of $\Re\left[E_{x}^{D}\right]$ for two different impact parameters $x_{0}=0$ and $40 \mu \mathrm{m}$. As can be seen, the field components' shape remains identical, the impact parameter is simply transformed into a spatial shift of the field in the detector plane. The direct shift implementation is true for the optical system magnification of $M=1$, for $M \neq 1$ it has simply to be scaled.

This finding has an important consequence comparing the case of incoherent and coherent OTR emission. In the first case it is the intensity following a universal dependency described by Eq. (4). In the case of coherent emission it is the field following a similar dependency. Therefore, in analogy to the case of incoherent emission the so-called field point-spread function (FPSF) is introduced which allows us to obtain the coherent sum of the OTR fields from all electrons inside a bunch by considering the single particle fields $E_{x, y}^{D}$ according to

$$
\begin{aligned}
& \operatorname{FPSF}_{x}\left(x_{D}-x_{0}\right)=E_{x}^{D}\left(x_{D}-x_{0}\right), \\
& \operatorname{FPSF}_{y}\left(y_{D}-y_{0}\right)=E_{y}^{D}\left(y_{D}-y_{0}\right) .
\end{aligned}
$$

The total field in the detector plane which is required in order to calculate the COTR pattern in the detector plane according to Eq. (7) can simply be derived from the convolution of the transverse beam distribution $\rho\left(x_{b}, y_{b}\right)$ of the electrons inside the bunch with the FPSF:

$$
\begin{aligned}
& E_{\text {coh }\{x, y\}}^{D}\left(x_{D}, y_{D}, \sigma_{x}, \sigma_{y}\right) \\
& =\operatorname{const} \int \mathrm{d} x_{b} \mathrm{~d} y_{b} \rho\left(x_{b}, y_{b}\right) \\
& \quad \times \operatorname{FPSF}_{\{x, y\}}\left(\left\{x_{D}-x_{b}\right\},\left\{y_{D}-y_{b}\right\}\right) .
\end{aligned}
$$

In this sense, the remaining four-fold integration which is required in order to determine the COTR field in the detector plane according to Eq. (6) is reduced to two twofold integrations: one for the convolution integral Eq. (10) which is numerically straightforward to solve, and one for the determination of the single particle OTR field Eq. (2).

In the following the expression for the latter one will further be simplified. The fact that dimensionless variables are used according to Eq. (1) and that the field components shape remains identical according to Fig. 5 suggests to introduce a kind of "universal" field shape. It will be used henceforth to describe the field $\Re\left[E_{x}^{D}\right]$ for any $x_{0}-$ coordinate by the dependence on the argument $x_{D}-x_{0}$. Hereinafter in the simulations $\Re\left[E_{x}^{D}\right]$ is approximated by an analytical expression with fitted parameters. As an example, $\Re\left[E_{x}^{D}\right]$ plotted in Fig. 5 is fitted by the function

$\operatorname{Re}\left[E_{x}^{D}\right]\left(x_{D}\right)=a_{0} x_{D} \exp \left\{-b_{0} x_{D}^{2}\right\}+\sum_{i=1}^{n} a_{k} \sin \left(b_{k} x_{D}\right)$.

The number of terms in Eq. (11) is determined by the required accuracy. In the present case the number was chosen as $n=10$ for a lens aperture $x_{m}=y_{m}=50$. The fit parameters for this case are summarized in Table I. The exponential term in Eq. (11) characterizes the "long-scale" behavior of the OTR field, the terms of the sum are determined by short period oscillations.

Figure 6 shows a comparison of $\Re\left[E_{x}^{D}\right]$ and $\operatorname{Re}\left[E_{x}^{D}\right]$ for a lens aperture of $x_{m}=y_{m}=50$. As can be concluded, the single particle OTR field Eq. (2) is well approximated by Eq. (11). Thus, in the present case the calculation of the COTR field in the detector plane is reduced to a convolution of the field approximation Eq. (11) and the transverse beam distribution $\rho\left(x_{b}, y_{b}\right)$.

It is instructive to consider the single particle OTR field also in the limiting case $X_{T_{\max }}, Y_{T_{\max }} \rightarrow \infty$ (or, in our approximation, $x_{T_{\max }}=y_{T_{\max }}=5$. In this situation a simple integral form can be derived, see, e.g., Ref. [17]. Following the literature, the field is usually expressed as radial polarized component instead of the Cartesian field components which were used throughout this work,

TABLE I. Eq. (11) terms.

\begin{tabular}{lccccccccccc}
\hline \hline$i$ & 0 & 1 & 2 & 3 & 4 & 5 & 6 & 7 & 8 & 9 & 10 \\
\hline$a_{i}$ & -3485.45 & -92.56 & -118.78 & 36.97 & 62.96 & 96.92 & -127.87 & 134.24 & 108.93 & -128.06 & 72.86 \\
$b_{i}$ & 26.35 & 15.06 & 34.56 & -9.96 & -3.36 & -44.10 & 19.85 & -24.70 & -39.42 & 29.63 & -48.27 \\
\hline \hline
\end{tabular}




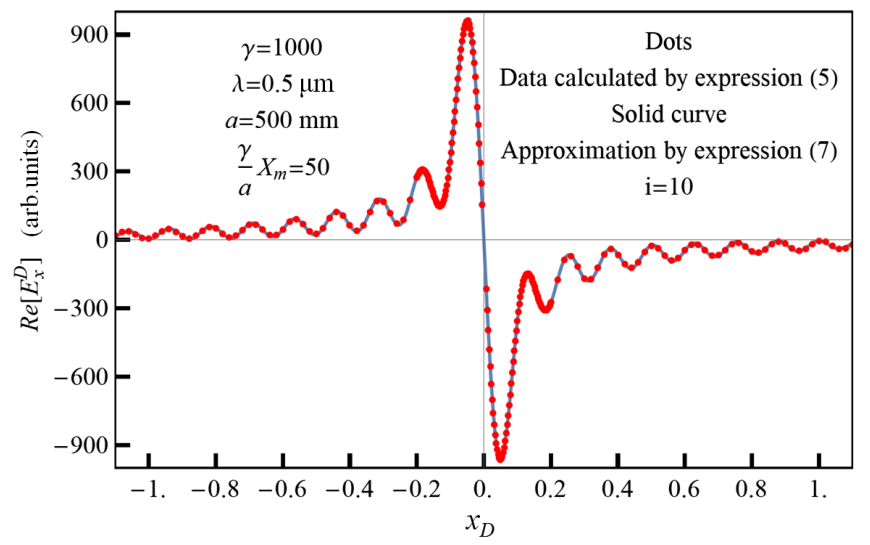

FIG. 6. Comparison of $\Re\left[E_{x}^{D}\right]$ (points) according to Eq. (2) with the approximation $\operatorname{Re}\left[E_{x}^{D}\right]$ (solid curve) given by Eq. (11) for a lens aperture of $x_{m}=y_{m}=50$.

$$
E_{r}^{D}\left(r_{D}\right)=\text { const } \int_{0}^{r_{m}} r_{L} /\left(1+r_{L}^{2}\right) \mathrm{J}_{1}\left(r_{L} r_{D}\right) r_{L} \mathrm{~d} r_{L}
$$

with $r_{i}=\sqrt{x_{i}^{2}+y_{i}^{2}}$ and the indices $i=L, D$ correspond to lens (L) and detector (D) plane as before and $r_{m}$ the aperture limit.

In case $r_{m} \gg 1$ for lens aperture, the integration in Eq. (12) can be performed analytically, resulting in [18]

$$
E_{r}^{D}\left(r_{D}\right)=\mathrm{const}\left\{\frac{1}{r_{D}}\left[r_{D} \mathrm{~K}_{1}\left(r_{D}\right)-\mathrm{J}_{0}\left(r_{D} r_{m}\right)\right]\right\} .
$$

Transforming Eq. (13) back into Cartesian components for convenience, the resulting fields are given by

$$
\begin{aligned}
& E_{\{x, y\}}^{D}\left(x_{D}, y_{D}, r_{m}\right) \\
& =\operatorname{const}\left\{-\frac{\left\{x_{D}, y_{D}\right\}}{x_{D}^{2}+y_{D}^{2}}\left[\sqrt{x_{D}^{2}+y_{D}^{2}} \mathrm{~K}_{1}\left(\sqrt{x_{D}^{2}+y_{D}^{2}}\right) \ldots\right.\right. \\
& \left.\left.\quad-\mathrm{J}_{0}\left(\sqrt{x_{D}^{2}+y_{D}^{2}} \sqrt{x_{m}^{2}+y_{m}^{2}}\right)\right]\right\} .
\end{aligned}
$$

The first "minus" sign in the curly brackets was introduced in order to allow a direct comparison between this analytical field approximation and the single particle OTR field Eq. (2). The result of this comparison is shown in Fig. 7. As can be seen, there is a reasonable coincidence between both distributions.

This approximation allows a direct determination of the two-dimensional FPSF with the fields

$$
\begin{aligned}
& \operatorname{FPSF}_{\{x, y\}}\left(x_{D}-x_{b}, y_{D}-y_{b}\right) \\
& \quad=E_{\{x, y\}}^{D}\left(x_{D}-x_{b}, y_{D}-y_{b}, r_{m}\right) .
\end{aligned}
$$

according to Eq. (14).
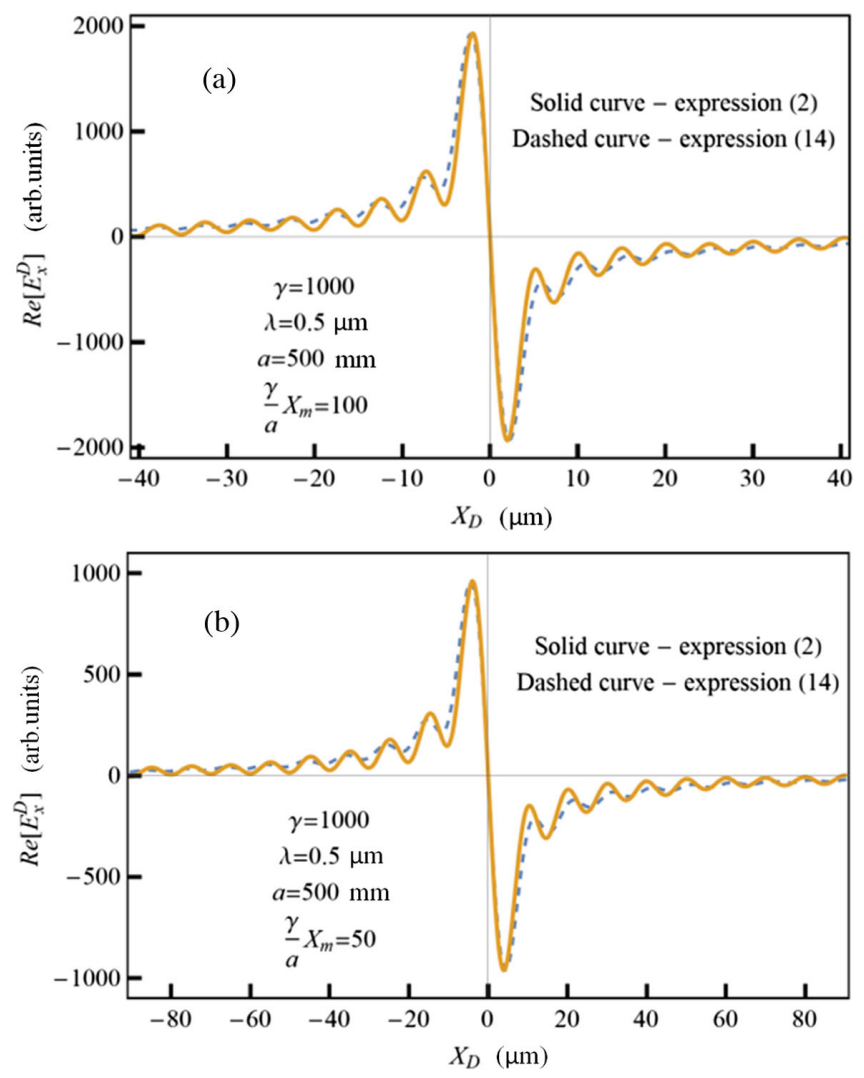

FIG. 7. Comparison of the analytical single particle OTR field approximation Eq. (14) and the exact expression Eq. (2) for two different lens apertures: (a) -100 , (b) -50 . The parameter set used for the calculation is shown in the insert.

\section{COTR IMAGE CALCULATION}

With knowledge of the FPSF it is possible to calculate an image of the bunch profile. Such a calculation should be performed taking into account both contributions from incoherent and coherent OTR fields. Performing the transition from a discrete electron bunch distribution to a continuous one [12], it is possible to derive the expression for both incoherent and coherent OTR intensity distributions in the detector plane from a bunch with $N$ particles, introducing a 2-dimensional Gaussian distribution for $\rho\left(x_{b}, y_{b}\right)$.

$$
\begin{aligned}
\frac{\mathrm{d}^{2} W_{\{x, y\}}^{B}}{\mathrm{~d} x_{D} \mathrm{~d} y_{D}}= & \text { const } \times N \exp \left\{-\frac{4 \pi^{2} \lambda^{2}}{\sigma_{z}^{2}}\right\} \\
& \times\left\{\int \mathrm{d} x_{b} \mathrm{~d} y_{b} \operatorname{PSF}\left(x_{D}-x_{b}, y_{D}-y_{b}\right)\right. \\
& \times \exp \left\{-\frac{x_{b}^{2}}{2 \sigma_{x}^{2}}-\frac{y_{b}^{2}}{2 \sigma_{y}^{2}}\right\}+(N-1) \\
& \times \mid \int \mathrm{d} x_{b} \mathrm{~d} y_{b} \operatorname{FPSF}_{\{x, y\}}\left(x_{D}-x_{b}, y_{D}-y_{b}\right) \\
& \left.\times\left.\exp \left\{-\frac{x_{b}^{2}}{2 \sigma_{x}^{2}}-\frac{y_{b}^{2}}{2 \sigma_{y}^{2}}\right\}\right|^{2}\right\} .
\end{aligned}
$$


Exploiting Eq. (16) allows to calculate the shape of a bunch image, depending on the rms bunch sizes $\sigma_{x}, \sigma_{y}$.

As a rule, $N \geq 10^{6}$ and it is sufficient to consider only the COTR contribution which will be done hereafter. For the sake of simplicity, in the following only the 1-dimensional dependence of the COTR image on the dimensionless transverse beam size $\sigma_{x}=\frac{2 \pi}{\gamma \lambda} \Sigma_{x}$ (with $\Sigma_{x}$ the rms size in micrometer) will be considered. In this case the integration of the coherent part in Eq. (16) reduces to

$E_{\mathrm{coh}}^{D}\left(x_{D}, \sigma_{x}\right)=\int \mathrm{d} x_{b} \frac{1}{\sqrt{2 \pi} \sigma_{x}} \exp \left\{-\frac{x_{b}^{2}}{2 \sigma_{x}^{2}}\right\} E_{x}^{D}\left(x_{D}-x_{b}\right)$.

Eq. (17) can be solved analytically using the field approximation Eq. (11). The result is

$$
\begin{aligned}
E_{\mathrm{coh}}^{D}\left(x_{D}, \sigma_{x}\right)= & \frac{a_{0} x_{D}}{\left(1+2 b_{0} \sigma_{x}^{2}\right)^{3 / 2}} \exp \left\{-\frac{x_{D}^{2}}{2\left(\frac{1}{2 b_{0}}+\sigma_{x}^{2}\right)}\right\} \\
& +\sum_{i=1}^{n} a_{k} \sin \left[b_{k} x_{D}\right] \exp \left\{-b_{k}^{2} \frac{\sigma_{x}^{2}}{2}\right\} .
\end{aligned}
$$

Figure 8 shows a comparison of calculated beam profile images for two different lens apertures. Besides the approximative field description Eq. (11), in addition the analytical approximation Eq. (14) was used. As can be seen, both approximations coincide in the range of the maxima, a slight discrepancy is observed only in the tails of the distributions.

The comparison in Fig. 8 indicates that the position of the maxima has only a very weak dependency on the size of the lens aperture (here: $x_{m}=50$ and $x_{m}=100$ ). This result is in contradiction with imaging using incoherent OTR where the beam profile image is determined by the PSF which strongly depends on the lens aperture [19].

In the present case the coherent FPSF distribution is also determined by the aperture. However, as result of the convolution with the bunch profile this dependency is lost. Bunch sizes considered here are much larger than the position of the fundamental maximum in FPSF and, as result, the pattern shape is predominantly determined by the bunch size. This fact is illustrated in Fig. 9 where the profile of a submicron bunch was calculated for both apertures $x_{m}=y_{m}=50$ and 100 .

In order to underline the insensitivity of the maximum position calculation on the field approximation in use, Fig. 10 shows a comparison for three different beam sizes $\sigma_{x}$. As mentioned before in Fig. 8, a slight discrepancy can be observed only in the tails of the distributions.
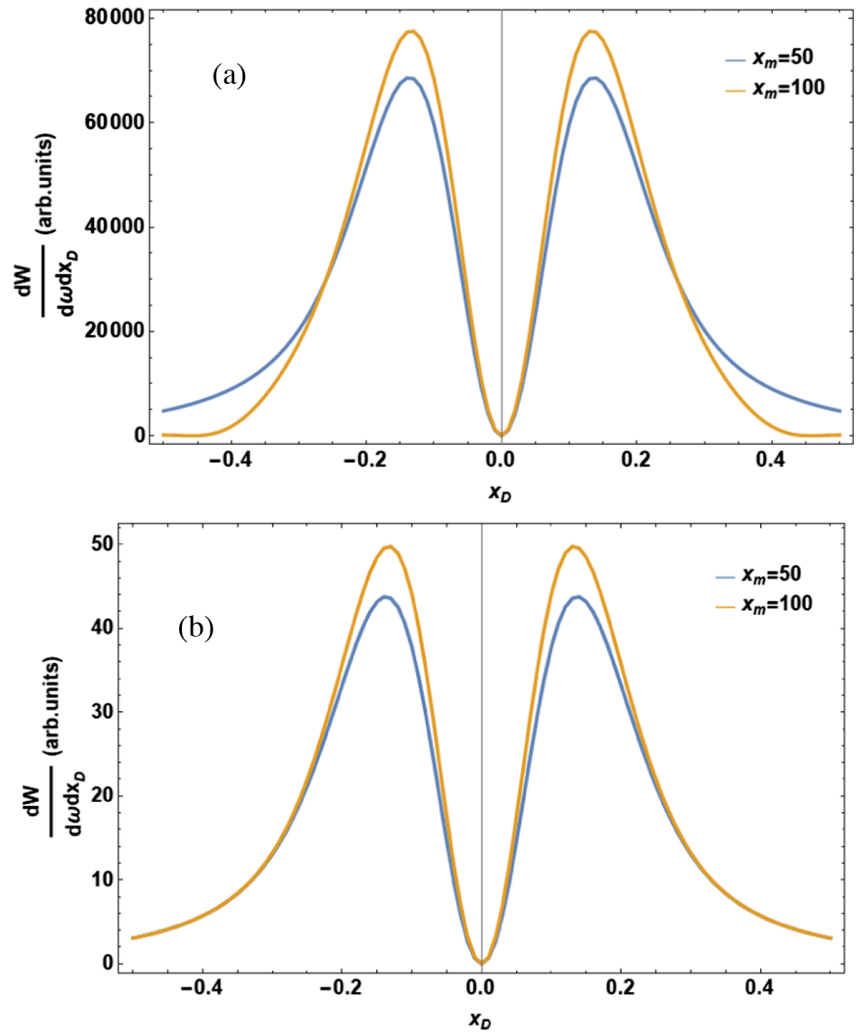

FIG. 8. Calculated COTR based beam profile images using the field approximations (a) Eq. (11) and (b) Eq. (14) for two different lens apertures. The dimensionless beam size amounts to $\sigma_{x}=0.1$, the remaining parameters are the same as before.

A similar calculation, but this time for dimensioned beam sizes $\Sigma_{x}=16,26$ and $40 \mu \mathrm{m}$ and two different beam energies $\gamma=1000$ and 2000 is plotted in Fig. 11. As can be seen, if the beam energy is doubled the maximum positions $X_{\max }$ change insignificantly, in the latter case there is only an increase of about 5\% in $X_{\max }$. Therefore the COTR pattern has only a rather weak dependency on the particle beam energy.

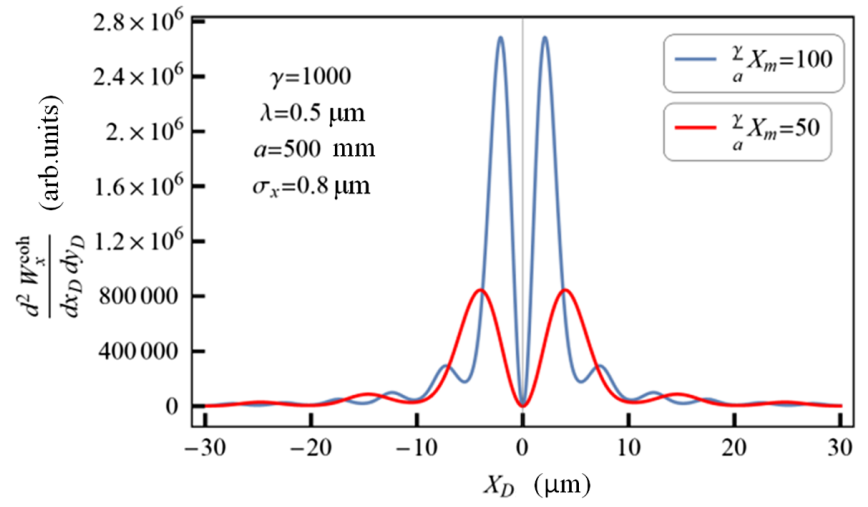

FIG. 9. Calculated COTR based beam profile image for a beam size of $\Sigma_{x}=0.8 \mu \mathrm{m}\left(\sigma_{x}=0.01\right)$ and two different lens apertures. The parameter set used for the calculation is shown in the insert. 


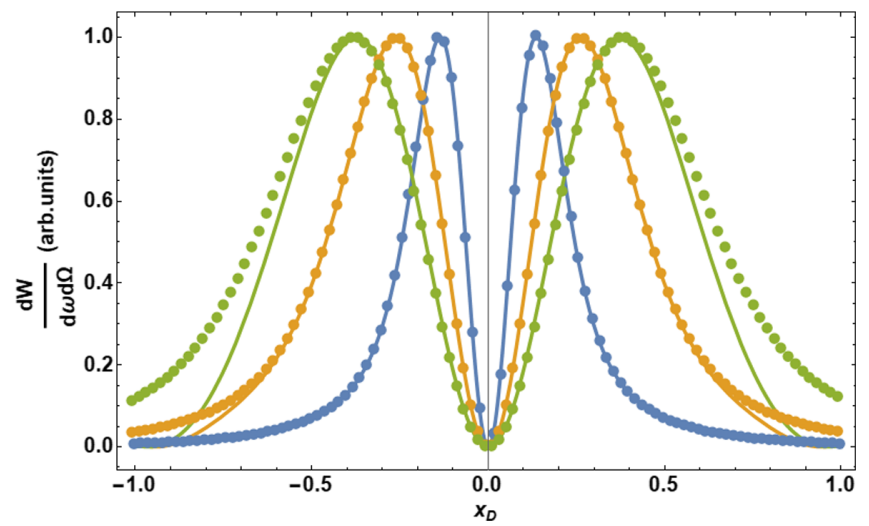

FIG. 10. Comparison of calculated COTR intensity distributions in the detector plane, using field approximations Eq. (11) (solid) and Eq. (14) (dashed) for different (dimensionless) beam sizes $\sigma_{x}=0.1$ (blue curve), 0.2 (orange) and 0.3 (green) and a lens aperture of $x_{m}=y_{m}=50$.

\section{CHARACTERISTICS OF THE COTR IMAGE}

In Fig. 12(a) the dependency of the maximum position in a COTR image as function of the beam size (for the interval
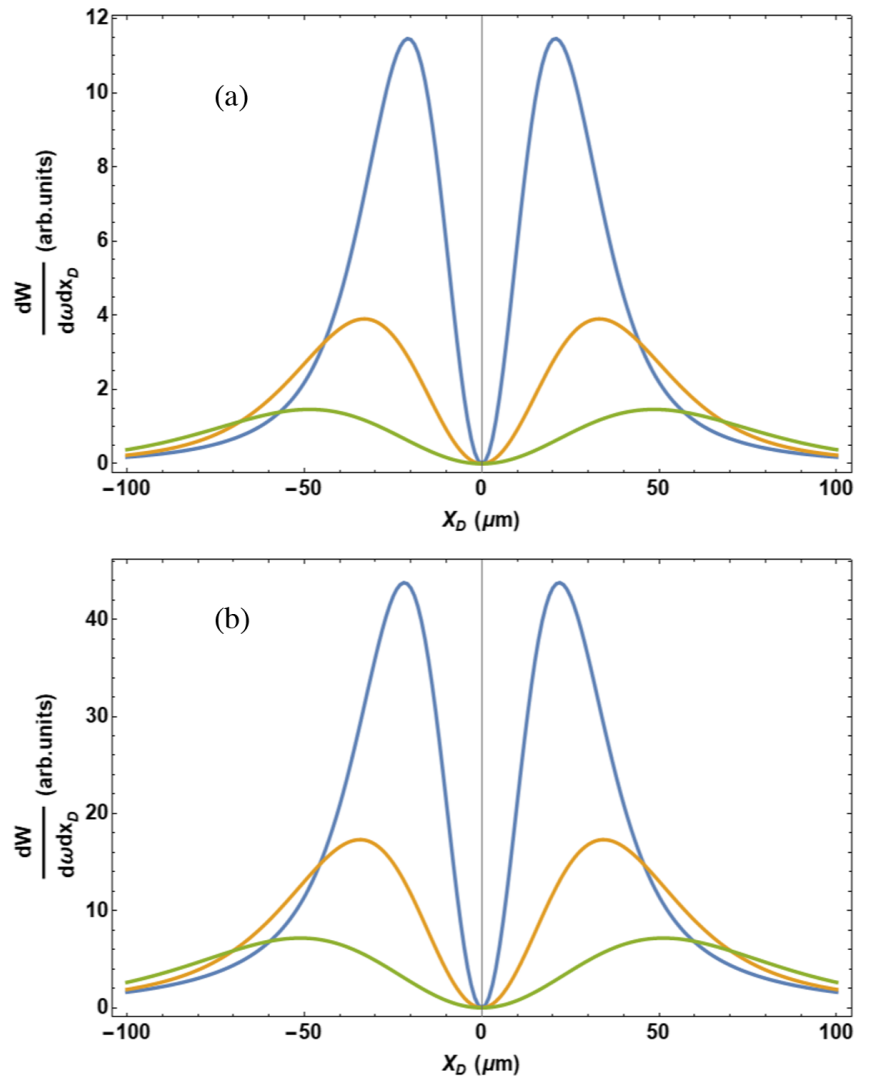

FIG. 11. Calculated COTR based beam profiles for a beam size of $\Sigma_{x}=16 \mu \mathrm{m}$ (blue), $26 \mu \mathrm{m}$ (yellow) and $40 \mu \mathrm{m}$ (green curve). The profiles are calculated for $\lambda=0.5 \mu \mathrm{m}$ based on approximation Eq. (11) and for two different beam energies $\gamma=1000$ (a) and $\gamma=2000(\mathrm{~b})$.
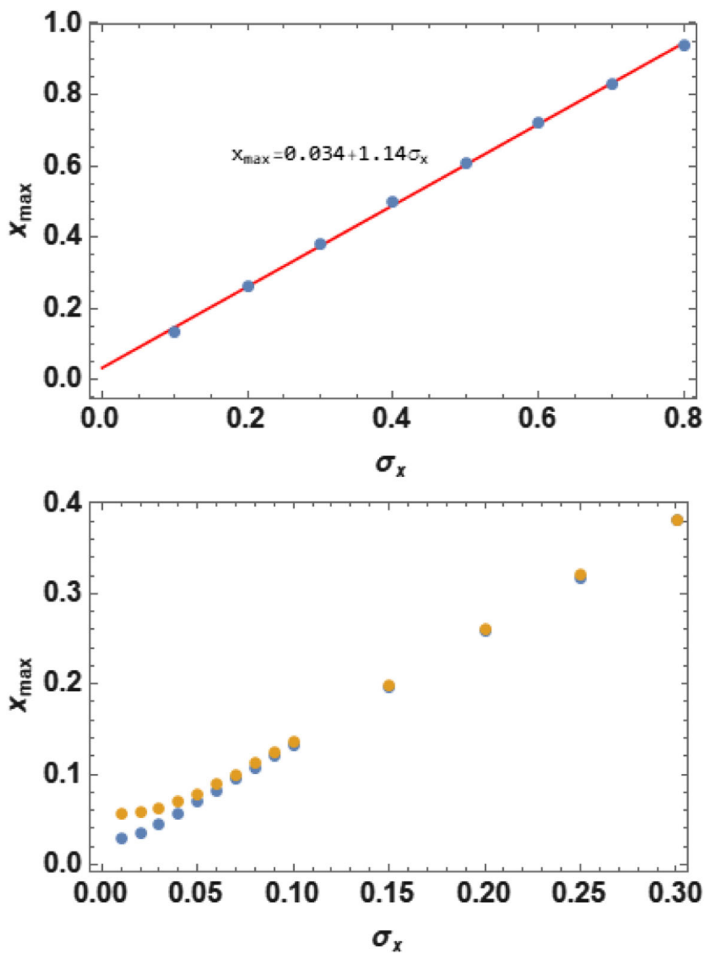

FIG. 12. Dependency of the maximums position $x_{m}$ on the beam size $\sigma_{x}$. Bottom one represents smaller beam size dependence. Lens aperture: $x_{m}=y_{m}=50$ (orange points); $x_{m}=y_{m}=$ 100 (blue points).

$\left.\sigma_{x} \geq 0.1\right)$ is shown in dimensionless coordinates. As can be seen, the maximum position $x_{\max }$ scales linearly with beam size and thus can be fitted using a linear regression, resulting in

$$
x_{\max }=0.034+1.14 \sigma_{x} .
$$

It is straightforward to rewrite Eq. (19) in dimensioned units:

$$
X_{\max }=0.0054 \gamma \lambda+1.14 \Sigma_{x} .
$$

In principle this equation allows us directly to estimate the beam size $\Sigma_{x}$ from the maximum $X_{m}$ of the measured COTR based image.

In order to give a rough estimate for the achievable resolution, Fig. 12(b) shows a similar calculation but for smaller beam sizes $\left(\sigma_{x \max } \leq 0.1\right)$. As expected, there is a dependency on the lens aperture (see also Fig. 9). This region of small beam sizes is dominated by the FPSF and imposes limitations on the spatial resolution. According to Fig. 12(b), the rough estimate is derived exploiting the deviation from the linear behavior which results in minimum values 

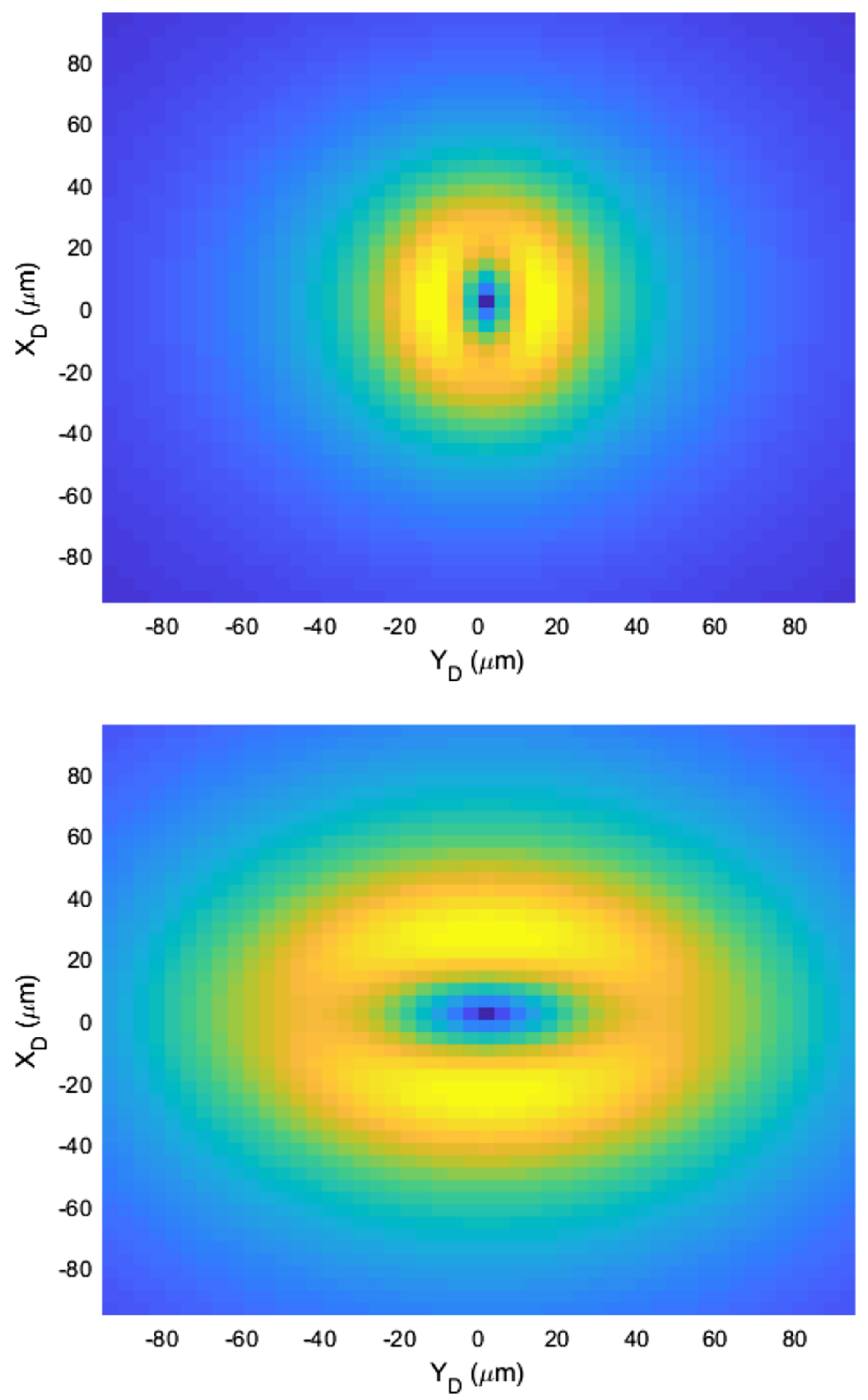

FIG. 13. 2-dimensional asymmetric COTR distributions, calculated for $\gamma=1000$ and $\lambda=0.5 \mu \mathrm{m}$. Top: beam sizes of $\Sigma_{x}=16 \mu \mathrm{m}, \Sigma_{y}=8 \mu \mathrm{m}$; bottom: $\Sigma_{x}=16 \mu \mathrm{m}, \Sigma_{y}=32 \mu \mathrm{m}$.

$$
\begin{aligned}
& \sigma_{x \min } \sim 0.03 \text { for } x_{m}=100 \\
& \sigma_{x \min } \sim 0.06 \text { for } x_{m}=50
\end{aligned}
$$

for the applicability of Eq. (19) resp. (20).

Using dimensioned units, it is possible to combine the results of Eq. (21) and rewrite it as function of the wavelength

$$
\Sigma_{x_{\min }} \sim \frac{\lambda}{2 \theta_{a p}}
$$

with $\theta_{a p}=\frac{X_{m}}{a}$ the lens aperture. As can be seen from Eq. (22), the resolution is determined only by $\lambda$ and $\theta_{a p}$, but in the ultrarelativistic approximation there is no dependency on $\gamma$.

For a lens with radius $50 \mathrm{~mm}$, placed at a distance $a=$ $500 \mathrm{~mm}$ (corresponding to $\theta_{a p}=0.1$ ) and observation
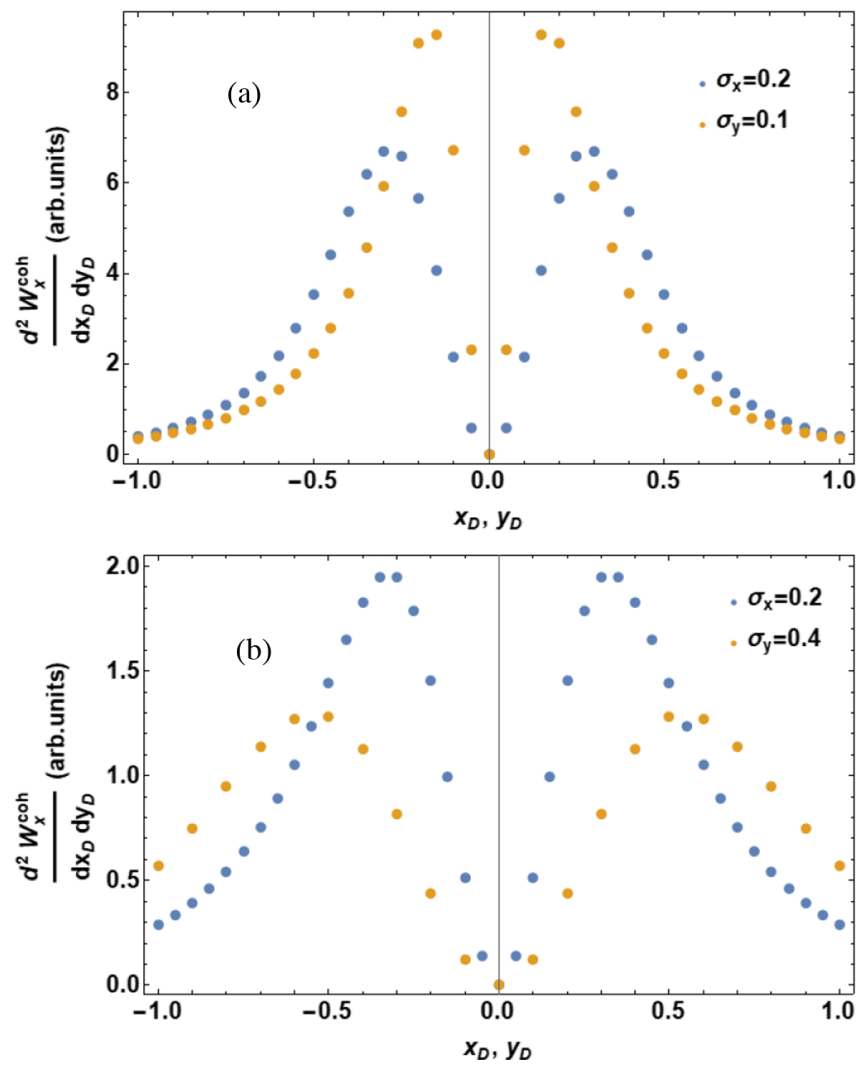

FIG. 14. Central cut through 2-dimensional asymmetric COTR distributions shown in Fig. 13: (a) corresopnds to the Fig. 13 top one, (b) corresponds to the bottom one. Yellow points correspond to the $y_{D}$ and blue ones to the $x_{D}$ distribution.

wavelength $\lambda=0.5 \mu \mathrm{m}$, the estimate Eq. (22) results in a minimum resolvable beam size of $\Sigma_{x_{\min }} \sim 2.5 \mu \mathrm{m}$.

Hereafter, an analogue characteristic as in Eq. (19) resp. (20) for the 2-dimensional case will be deduced. Due to the findings that (i) beam size information is extracted from the maximum position $\{x, y\}_{\max }$, and that (ii) the 2-dimensional analytical field approximation Eq. (14) describes the maximum position with high accuracy (cf. Figs. 8 and 10), the following calculations are based on this approximation. Distributions are calculated along the axes $y_{D}=0$ and $x_{D}=0$ using the corresponding polarization direction according to Eqs. (14) and (15).

For asymmetric bunches $\sigma_{x} \neq \sigma_{y}$, the corresponding distributions should carefully be distinguished. Figures 13 and 14 show examples with reverted maximum positions for $\mathrm{x}$ - and $\mathrm{y}$-direction. The (dimensionless) beam sizes in these examples amount to (a) $\sigma_{x}=0.2, \sigma_{y}=0.1$ and (b) $\sigma_{x}=0.2, \sigma_{y}=0.4$.

It should be noted that the COTR intensity depends not only on the bunch length but also on the bunch transverse size as can be seen from Fig. 15. Such dependency is described by the transverse form factor (see, e.g., Ref. [12]): 


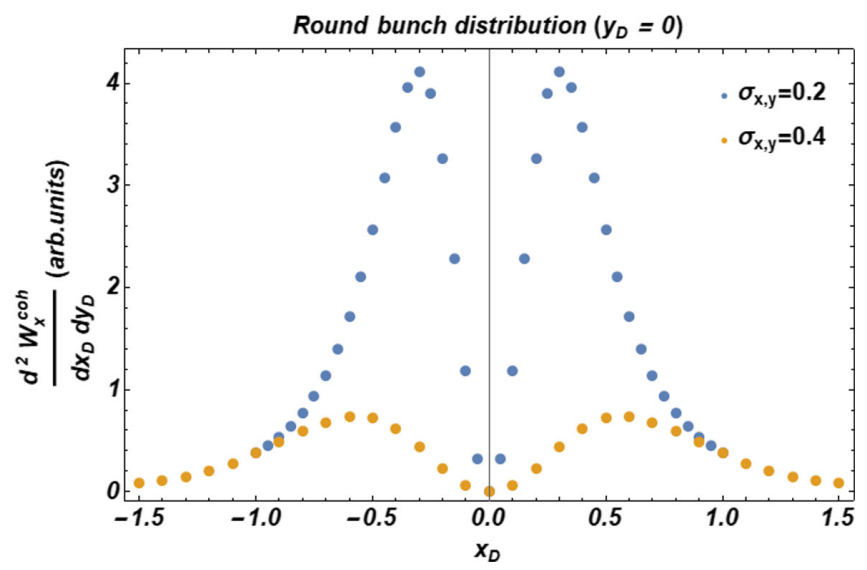

FIG. 15. COTR distribution calculated for an azimuthally symmetric bunch.

$$
\begin{aligned}
F_{\perp} & =\int \mathrm{d} x \mathrm{~d} y \exp \left\{-i\left(k_{x} x+k_{y} y\right)\right\} \rho(x, y) \ldots \\
& =\exp \left\{-\frac{1}{2}\left(\frac{2 \pi \sin \theta}{\lambda}\right)^{2}\left(\sigma_{x}^{2} \cos ^{2} \varphi+\sigma_{y}^{2} \sin ^{2} \varphi\right)\right\}
\end{aligned}
$$

with $\theta, \varphi$ the polar resp. azimuthal angles characterizing the wave vector of the emitted photon.

For such azimuthal symmetric bunch the position of the maximum is described by a linear dependency with an increased coefficient compared to the case under consideration in Eq. (19): $r_{m}=0.027+1.36 \sigma$.

\section{DISCUSSION AND CONCLUSION}

The authors of Ref. [15] described a COTR profile in the image plane by the expression

$$
I_{\mathrm{coh}} \sim\left|\partial_{x} \rho_{\perp}\left(x_{D}, y_{D}\right)\right|^{2}+\left|\partial_{y} \rho_{\perp}\left(x_{D}, y_{D}\right)\right|^{2} .
$$

For a Gaussian bunch profile, the image would therefore be described again by a Gaussian

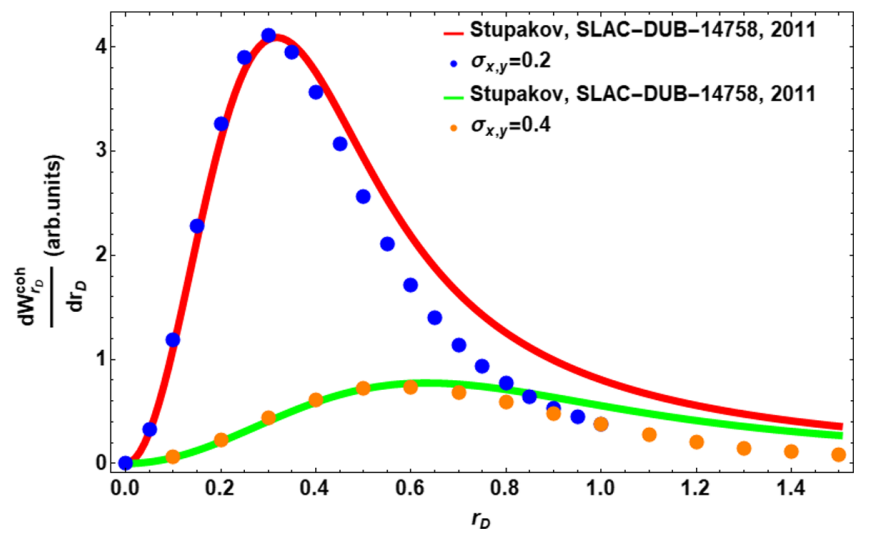

FIG. 16. COTR based image calculated for an azimuthal symmetric bunch. In addition, the results of Ref. [21] are plotted.

$$
I_{\mathrm{coh}} \sim\left(\frac{x_{D}^{2}}{\sigma_{x}^{4}}+\frac{y_{D}^{2}}{\sigma_{y}^{4}}\right) \exp \left\{-\frac{x_{D}^{2}}{\sigma_{x}^{2}}\right\} \exp \left\{-\frac{y_{D}^{2}}{\sigma_{y}^{2}}\right\}
$$

For a round bunch this distribution possesses a maximum at $r_{D}=\sigma$ which is smaller than the simulation result of the present work (see Fig. 15).

Furthermore, the author of Ref. [20] calculated a COTR based image for a round beam. He obtained an intensity distribution in the detector plane with a maximum at $r_{D} \approx 1.6 \sigma$ which is close to our calculations, see Fig. 16 .

It should be noted that Eq. (19) is valid only for the distribution along the major axis of the bunch profile, i.e., the condition $\sigma_{x}^{2} \gg \sigma_{y}^{2}$ has to be fulfilled. For a round bunch $\left(\sigma_{x}=\sigma_{y}\right)$ the distribution along $y_{D}$ (or $x_{D}$ ) may be considered as radial distribution of the image, see Fig. 15. For such azimuthal symmetric bunch the position of the maximum is described by a linear dependence with an increased coefficient compared to the case under consideration in Eq. (19): $r_{m}=0.027+1.36 \sigma$.

The approach presented in this work allows to perform a quantitative analysis of bunch profiles measured with COTR and a focusing lens. Expressions were obtained for both polarization components $E_{x}, E_{y}$ of the COTR field. In the model, a finite lens aperture was taken into account.

The propagation of COTR through an optical system was simulated as convolution of the COTR field in the image plane with a distribution describing the transverse beam profile (in contrast to incoherent OTR where such convolution is performed with the intensity but not with the field). The field components $E_{x}, E_{y}$ described, e.g., by Eq. (14) both depend on the coordinates $x_{D}, y_{D}$ and can easily be applied for a convolution with any asymmetric bunch profile.

For a bunch with Gaussian transverse beam profile, images were calculated assuming a conventional optical system. In principle, the task of field propagation through an optical system could also be solved by a commercial program like Zemax [7], using the known COTR fields (in analogy to Zemax calculations for incoherent OTR, see Refs. [21,22]).

It was shown that the resulting distributions are characterized by a central minimum along the beam direction, and that the position of the intensity maxima is predominantly influenced by the transverse bunch size.

Equation (20) which connects a measured $X_{\max }$ value with the beam size $\Sigma_{x}$ depends weakly on the Lorentz factor $\gamma$. For the case under consideration $(\gamma=1000$, $\lambda=0.5 \mu \mathrm{m})$, an energy spread of the initial beam around $20 \%$ leads to $X_{\max }$ variations smaller than $1 \%$ for a beam size of $\Sigma_{x} \geq 10 \mu \mathrm{m}$.

For the special case of a round bunch profile there exists a radial polarization component from which it is possible to derive the Cartesian components $E_{x}$ and $E_{y}$.

In the experiment described in Ref. [23] the authors observed COTR interference fringes from an 
ultrarelativistic electron bunch $(E=215 \mathrm{MeV})$ using two OTR screens. Such a bunch generated by the mechanism of laser-plasma acceleration possesses a strong asymmetry in the $x-y$ plane. The approach described above can be applied for simulation of COTR characteristics from such kind of bunches.

In the authors' previous paper Ref. [24] a method was proposed to improve the spatial resolution of an OTR transverse beam profile monitor using the scheme of asymmetric light collection of incoherent OTR. The same approach can in principle be used for the case of COTR and will be subject of future investigations.

\section{ACKNOWLEDGMENTS}

This work was partly supported by the program "Nauka" of the Russian Ministry of Science, Grant No. FSWW2020-0008 and by the TPU Competitiveness Enhancement Program.

[1] X. Artru, R. Chehab, K. Honkavaara, and A. Variola, Resolution power of optical transition radiation. Theoretical considerations, Nucl. Instrum. Methods Phys. Res., Sect. B 145, 160 (1998).

[2] A. H. Lumpkin, B. X. Yang, W. J. Berg, M. White, J. W. Lewellen, and S. V. Milton, Optical techniques for electron-beam characterizations on the APS SASE FEL project, Nucl. Instrum. Methods Phys. Res., Sect. A 429, 336 (1999).

[3] M. Ross, S. Andersen, J. Frish et al., A very high resolution optical transition radiation beam profile monitor, Report No. SLAC-PUB-9280, 2002.

[4] A. Cianchi, M. Castellano, L. Catani, E. Chiadroni, K. Honkavaara, and G. Kube, Nonintercepting electron beam size monitor using optical diffraction radiation interference, Phys. Rev. Accel. Beams 14, 102803 (2011).

[5] P. Karataev, A. Aryshev, S. Boogert, D. Howell, N. Terunuma, and J. Urakawa, First Observation of the Point Spread Function of Optical Transition Radiation, Phys. Rev. Lett. 107, 174801 (2011).

[6] J. Wolfenden, R. B. Fiorito, and C. P. Welsch, A novel simulation and analysics algorithm for high resolution optical transition radiation imaging, Opt. Express 27, 2988 (2019).

[7] Zemax Optic Studio 16.5, Users Manual, Zemax, 2017.

[8] W. P. Leemans, C. G. R. Geddes, J. Faure et al., Observation of Terahertz Emission from a Laser-Plasma Accelerated Electron Bunch Crossing a Plasma-Vacuum Boundary, Phys. Rev. Lett. 91, 074802 (2003).

[9] E. Saldin, E. Schneidmiller, and M. Yurkov, The Physics of Free Electron Lasers (Springer-Verlag, Berlin, 2010).

[10] U. Dorda, R. Assmann, R. Brinkmann et al., SINBAD-The accelerator R\& D facility under construction at DESY, Nucl. Instrum. Methods Phys. Res., Sect. A 829, 233 (2016).
[11] M. C. Downer, R. Zgadzaj, A. Debus, U. Schramm, and M. C. Kaluza, Diagnostics for plasma-based electron accelerators, Rev. Mod. Phys. 90, 035002 (2018).

[12] C. B. Schroeder, E. Esarey, J. van Tilborg, and W. P. Leemans, Theory of coherent transition radiation generated at a plasma-vacuum interface, Phys. Rev. E 69, 016501 (2004).

[13] H. Loos, R. Akre, A. Brachmann, F.-J. Decker et al., Observation of coherent optical transition radiation in the LCLS Linac, Report No. SLAC-PUB-13395, 2008.

[14] R. B. Fiorito, M. Cornacchia, and A. G. Shkvarunets, Simulation of coherent optical transition radiation in linac based free electron lasers, in Proceedings of FEL 2009, 356 Liverpool, UK (2009), https://accelconf.web.cern.ch/ FEL2009/papers/tupc49.pdf.

[15] N. Bourgeois, M. Heigoldt, W. Rittershofer et al., Transverse beam profile measurements of laser accelerated electrons using coherent optical radiation, AIP Conf. Proc. 1507, 258 (2012).

[16] G. Geloni, P. Ilinski, E. Saldin, E. Schneidmiller, and M. Yurkov, Coherent optical transition radiation as a tool for ultrashort electron bunch diagnostics, in Proceedings of the 9th European Workshop on Beam Diagnostics and Instrumentation for Particle Accelerators of DIPAC 2009, (Basel, Switzerland 2009), p. 251, https://accelconf.web .cern.ch/d09/papers/tupb37.pdf.

[17] A. P. Potylitsyn, Advanced Radiation Sources and Applications (NATO Science Series II, Springer, N.Y., 2006), pp. $199,149$.

[18] D. Xiang, W.-H. Huang, and Y.-Z. Lui, Imaging of highenergy electron beam profile with optical diffraction radiation, Phys. Rev. Accel. Beams 10, 062801 (2007).

[19] G. Kube, Imaging with optical transition radiation, transverse beam diagnostics for the XFEL, TESLA-FEL Report No. TESLA-FEL 2008-01, 2008.

[20] G. Stupakov, Image formation by incoherent and coherent transition radiation from flat and rough surfaces, SLAC National Accelearator Laboratory Report No. 14758, 2011.

[21] F. G. Bisesto, M. Castellano, E. Chiadroni, and A. Cianci, Zemax simulation describing collective effects in transition and diffraction radiation, Opt. Express 26, 5075 (2018).

[22] T. Aumeyr, M. G. Billing, L. M. Bobb, B. Bolzon, E. Bravin, P. Karataev, K. Kruchinin, T. Lefevre, and S. Mazzoni, Advanced simulations of optical transition and diffraction radiation, Phys. Rev. Accel. Beams 18, 042801 (2015).

[23] A. Lumpkin, M. LaBerge, D. Rule et al., Observations of coherent optical transition radiation interference fringes generated by laser plasma accelerator electron beamlets, FERMILAB Report No. FERMILAB-CONF-18-511-AD, 2018.

[24] A. Potylitsyn, L. Sukhikh, G. Kube, and A. Novokshonov, Spatial resolution improvement for an optical transition radiation monitor by asymmetric light collection, Opt. Express 26, 30231 (2018). 